\title{
Maternal fat consumption during pregnancy and risk of wheeze and eczema in Japanese infants aged 16-24 months: the Osaka Maternal and Child Health Study
}

\author{
Y Miyake, ${ }^{1}$ S Sasaki, ${ }^{2}$ K Tanaka, ${ }^{1}$ S Ohfuji, ${ }^{3}$ Y Hirota $^{3}$
}

\begin{abstract}
${ }^{1}$ Department of Public Health, Faculty of Medicine, Fukuoka University, Fukuoka, Japan; ${ }^{2}$ Department of Social and Preventive Epidemiology, School of Public Health, The University of Tokyo, Tokyo, Japan; ${ }^{3}$ Department of Public Health, Osaka City University Graduate School of Medicine, Osaka, Japan
\end{abstract}

Correspondence to: Dr Y Miyake, Department of Public Health, Faculty of

Medicine, Fukuoka University, Fukuoka 814-0180, Japan; miyake-y@fukuoka-u.ac.jp

Received 1 March 2009 Accepted 25 May 2009

Published Online First

3 June 2009

\begin{abstract}
Background: One factor capable of modulating antenatal immune responses is diet. This prospective study examined the association between maternal intake of specific types of fatty acids, cholesterol, fish and meat during pregnancy and the risk of wheeze and eczema in the offspring.
\end{abstract}

Methods: Subjects were 763 mother-child pairs. Data on maternal intake during pregnancy were assessed with a diet history questionnaire. Data on wheeze and eczema based on criteria of the International Study of Asthma and Allergies in Childhood were obtained from a questionnaire completed by mothers 16-24 months postpartum.

Results: Higher maternal intake of $\alpha$-linolenic acid and docosahexaenoic acid during pregnancy was independently associated with a reduced risk of wheeze, but not eczema, in the offspring (adjusted odds ratios (ORs) between extreme quartiles 0.52 (95\% $\mathrm{Cl} 0.28$ to 0.97$)$ and 0.37 (95\% Cl 0.15 to 0.91), respectively). Higher maternal intake of $n-6$ polyunsaturated fatty acids and linoleic acid during pregnancy was independently related to an increased risk of infantile eczema but not wheeze (adjusted ORs 2.25 (95\% Cl 1.13 to 4.54) and 2.11 (95\% Cl 1.06 to 4.26), respectively). No significant exposureresponse relationships were observed between maternal consumption of total fat, saturated fatty acids, monounsaturated fatty acids, eicosapentaenoic acid, arachidonic acid, cholesterol, meat and fish and the ratio of $n-3$ to $n-6$ polyunsaturated fatty acid consumption during pregnancy and infantile wheeze or eczema.

Conclusions: Maternal intake of $\alpha$-linolenic acid and docosahexaenoic acid during pregnancy may be preventive against infantile wheeze. Maternal intake of $n-6$ polyunsaturated fatty acids, especially linoleic acid, during pregnancy may increase the risk of childhood eczema.

One of the factors capable of modulating antenatal immune responses is diet. In particular, $n-3$ and n- 6 polyunsaturated fatty acids can influence the Th1 and Th2 balance. ${ }^{1}$ A case-control study of US schoolchildren showed that maternal oily fish consumption at least monthly versus no consumption during pregnancy was significantly related to a decreased risk of early persistent asthma in the children. ${ }^{2}$ A significant preventive relationship was observed between maternal fish intake during pregnancy and the risk of atopic wheeze in the offspring at 6 years of age in a Spanish prospective cohort study. ${ }^{3}$ However, a cohort study in the Netherlands reported no association between maternal fish intake during pregnancy and the risk of wheeze in children from 1 to 8 years of age. ${ }^{4}$ In cohort studies in Spain, Germany and the UK, a protective relationship was found between maternal fish intake during pregnancy and the risk of infantile eczema. ${ }^{3} 6$ The cohort study in Germany also showed significant positive associations of maternal intake of margarine and vegetable oils during pregnancy with the risk of eczema during the first 2 years in children. ${ }^{5}$

Our previous preliminary report found that higher maternal meat intake during pregnancy was significantly associated with an increased risk of suspected atopic eczema, which was based on a physician's diagnosis of atopic eczema or possible atopic eczema, in infants aged 3-4 months.' Using data from the 16-24-month follow-up of the cohort with definitions of wheeze and eczema according to the diagnostic criteria of the International Study of Asthma and Allergies in Childhood (ISAAC), we gained further insights into the relation between maternal intake of specific types of fatty acids, cholesterol, meat and fish during pregnancy and the risk of wheeze and eczema in infants in Japan where fish intake is high.

\section{METHODS}

\section{Study population}

The Osaka Maternal and Child Health Study (OMCHS) is a prospective cohort study. Details of the OMCHS have been described elsewhere. ${ }^{8} \mathrm{We}$ recruited pregnant women at baseline. Neyagawa City is one of the 43 municipalities in Osaka Prefecture, a metropolis in Japan. Of the 3639 eligible subjects in Neyagawa City, 627 women $(17.2 \%)$ participated in the OMCHS between November 2001 and March 2003. In order to increase the sample size, pregnant women living in municipalities other than Neyagawa City were also recruited. Eight pregnant women who did not live in Neyagawa City but who had become aware of the OMCHS at an obstetric clinic before August 2002 decided by themselves to participate in the OMCHS. In addition, there were 77 participants who received details of the OMCHS from public health nurses in six other municipalities from August 2002 to March 2003. From October 2002 to March 2003, 290 participants were recruited from a university hospital and three obstetric hospitals in three other municipalities.

A total of 1002 pregnant women at any stage of pregnancy gave their fully informed consent in 
writing and completed the baseline survey. Of the 1002 subjects, 867 mother-child pairs participated in the second survey from 2 to 9 months postpartum. Of the 867 motherchild pairs, 763 participated in the third survey from 16 to 24 months postpartum. The population analysed consisted of these 763 mother-child pairs.

\section{Measurements}

At baseline, each participant filled out a set of two selfadministered questionnaires. Also, a self-administered questionnaire was used in both the second and third survey. Participants mailed these materials to the data management centre at the time of each survey. Research technicians completed missing or illogical data by telephone interview.

In the baseline survey, dietary habits during the preceding month were assessed using a self-administered, semi-quantitative, comprehensive diet history questionnaire (DHQ). Details of the structure of the $\mathrm{DHO}$, calculation of dietary intake and validity of commonly studied nutritional factors have been published elsewhere. ${ }^{910}$ Estimates of daily intake for foods (150 items in total), energy and selected nutrients were calculated using an ad hoc computer algorithm for the $\mathrm{DHQ}^{9}{ }^{10}$ based on the Standard Tables of Food Composition in Japan. ${ }^{11}{ }^{12}$ Information on dietary supplements was not used in the calculation of dietary intake. The correlation coefficients for nutrient intake between those estimated from the $\mathrm{DHO}$ and those observed by a 3-day dietary record were $0.75,0.50,0.37$ and 0.49 for saturated fatty acids, monounsaturated fatty acids, polyunsaturated fatty acids and cholesterol, respectively, in women. ${ }^{9}$ A highly positive correlation was also observed between intake of $n-3$ polyunsaturated fatty acids of marine origin estimated by the $\mathrm{DHO}$ and the corresponding concentration in the serum phospholipid fraction in women $(r=0.69) .{ }^{10}$ Energy-adjusted intake by the residual method was used for the analyses. ${ }^{13}$

A second questionnaire at baseline asked about maternal age, gestation, family income, maternal and paternal education, maternal and paternal history of asthma, atopic eczema and allergic rhinitis and changes in diet in the previous month. A paternal or maternal history of asthma, atopic eczema and allergic rhinitis was defined as positive if the respective parent had been treated with medications for any of these allergic disorders at some time before the start of the survey.

A self-administered questionnaire in the second survey elicited information on the sex, birth weight and date of birth of the baby, the number of older siblings, maternal smoking during pregnancy and smoking in the household. A selfadministered questionnaire in the third survey included questions on breastfeeding duration in months and symptoms of wheeze and eczema based on the ISAAC phase I questionnaire. ${ }^{14} 15$ Wheeze was defined as present if the mother answered "yes" to the written question: "Has your child had wheezing or whistling in the chest in the last 12 months?". For eczema, affirmative answers to the following three questions were required: "Has your child ever had an itchy rash which was coming and going for at least 6 months?", "Has your child had this itchy rash at any time in the last 12 months?" and "Has this itchy rash at any time affected any of the following places: the folds of the elbow, behind the knees, in front of the ankles, under the buttocks, or around the neck, ears, or eyes?".

\section{Statistical analysis}

The intake of dietary factors under investigation was categorised at quartile points based on the distribution in 763 subjects. The following variables were a priori selected as potential confounding factors: maternal age, gestation at baseline, residential municipality, family income, maternal and paternal education, maternal and paternal history of asthma, atopic eczema and allergic rhinitis, maternal intake of vitamins $\mathrm{D}$ and $\mathrm{E}$ during pregnancy, changes in maternal diet in the previous 1 month, season when data at baseline were collected, maternal smoking during pregnancy, baby's older siblings, baby's sex, baby's birth weight, household smoking in the same room as the infant, breastfeeding duration and time of delivery before the third survey.

Logistic regression analysis was used to estimate crude odds ratios (ORs) and 95\% confidence intervals (CIs) of wheeze and eczema for each category of dietary intake under study in comparison with the lowest intake category. Multiple logistic regression analysis was employed to control for potential confounding factors. Trend of association was assessed by a logistic regression model assigning consecutive integers (1-4) to the quartiles of the exposure variables. All statistical analyses were conducted using the SAS software package Version 9.1 (SAS Institute, Cary, North Carolina, USA).

\section{RESULTS}

Of the 763 infants, the third survey revealed that 169 (22.1\%) and $142(18.6 \%)$ had symptoms of wheeze and eczema based on the ISAAC criteria, respectively. About $75 \%$ of infants had been breastfed for 6 months or longer (table 1). Slight or substantial changes in diet in the previous month were reported by 551 mothers due to nausea gravidarum $(n=453)$, maternal and fetal health $(n=89)$ and other reasons $(n=9)$. Maternal mean daily total energy intake and energy-adjusted consumption of $n-3$ and $\mathrm{n}-6$ polyunsaturated fatty acids during pregnancy were $7629 \mathrm{~kJ}$, $2.4 \mathrm{~g}$ and $11.5 \mathrm{~g}$, respectively (table 2 ).

Table 3 presents ORs and 95\% CIs for the risk of wheeze and eczema in the offspring according to maternal dietary intake of specific types of fatty acids and cholesterol during pregnancy. After adjustment for the confounding factors under study, the maternal intake of total fat, saturated fatty acids, monounsaturated fatty acids and n-3 polyunsaturated fatty acids during pregnancy was not measurably related to the risk of wheeze or eczema in the offspring. Compared with maternal $\alpha$-linolenic acid intake during pregnancy in the first quartile, such intake in the fourth quartile was independently associated with a reduced risk of wheeze in the offspring (adjusted OR 0.52, 95\% CI 0.28 to 0.97 ), although the inverse exposure-response relationship was not statistically significant. There was no association between maternal $\alpha$-linolenic acid intake during pregnancy and the risk of infantile eczema. Maternal eicosapentaenoic acid intake during pregnancy was not related to the risk of childhood wheeze or eczema. Maternal consumption of docosahexaenoic acid in the second and fourth quartiles was independently related to a decreased risk of wheeze in the infants, although the inverse linear trend was not statistically significant (adjusted OR between extreme quartiles $0.37,95 \%$ CI 0.15 to 0.91 ). No such relationship was detected for infantile eczema. Maternal intake of n-6 polyunsaturated fatty acids and linoleic acid during pregnancy in the highest quartile was independently associated with an increased risk of infantile eczema, but not wheeze, showing clear inverse exposure-response relationships (adjusted ORs 2.25 (95\% CI 1.13 to 4.54) and 2.11 (95\% CI 1.06 to 4.26), $\mathrm{p}$ for trend $=0.01$ and 0.03 , respectively). No statistically significant exposure-response relationships were observed between maternal intake of arachidonic acid and cholesterol and the ratio of $n-3$ to $n-6$ polyunsaturated fatty 
Table 1 Distribution of selected characteristics of 763 parent-child pairs

\begin{tabular}{|c|c|}
\hline Variable & No $(\%)$ or mean (SD) \\
\hline \multicolumn{2}{|l|}{ Baseline characteristics } \\
\hline Maternal age (years) & $30.0(4.0)$ \\
\hline Gestation (weeks) & $17.7(6.7)$ \\
\hline \multicolumn{2}{|l|}{ Residential municipality } \\
\hline Neyagawa City & $457(59.9)$ \\
\hline Other than Neyagawa City & $306(40.1)$ \\
\hline \multicolumn{2}{|l|}{ Family income (\% yen/year) } \\
\hline$<4000000$ & $211(27.7)$ \\
\hline $4000000-5999999$ & $313(41.0)$ \\
\hline $6000000+$ & $239(31.3)$ \\
\hline \multicolumn{2}{|l|}{ Maternal education (\% years) } \\
\hline$<13$ & $212(27.8)$ \\
\hline $13-14$ & $329(43.1)$ \\
\hline$\geqslant 15$ & $222(29.1)$ \\
\hline \multicolumn{2}{|l|}{ Paternal education (\% years) } \\
\hline$<13$ & $289(37.9)$ \\
\hline $13-14$ & $127(16.6)$ \\
\hline$\geqslant 15$ & $347(45.5)$ \\
\hline Maternal history of asthma (\%) & $80(10.5)$ \\
\hline Maternal history of atopic eczema (\%) & $120(15.7)$ \\
\hline Maternal history of allergic rhinitis (\%) & $256(33.6)$ \\
\hline Paternal history of asthma (\%) & $64(8.4)$ \\
\hline Paternal history of atopic eczema (\%) & $72(9.4)$ \\
\hline Paternal history of allergic rhinitis (\%) & $143(18.7)$ \\
\hline \multicolumn{2}{|l|}{ Changes in diet in the previous month (\%) } \\
\hline None or seldom & $212(27.8)$ \\
\hline Slight & $336(44.0)$ \\
\hline Substantial & $215(28.2)$ \\
\hline \multicolumn{2}{|l|}{ Season when data were collected (\%) } \\
\hline Spring & $241(31.6)$ \\
\hline Summer & $127(16.6)$ \\
\hline Fall & $167(21.9)$ \\
\hline Winter & $228(29.9)$ \\
\hline \multicolumn{2}{|l|}{ Characteristics at postnatal assessment } \\
\hline Maternal smoking during pregnancy (\%) & $97(12.7)$ \\
\hline Baby's older siblings $(\% \geqslant 1)$ & $381(49.9)$ \\
\hline Baby's sex (\% male) & $403(52.8)$ \\
\hline Baby's birth weight (g) & $3071.0(415.5)$ \\
\hline Household smoking in same room as infant & $195(25.6)$ \\
\hline \multicolumn{2}{|l|}{ Breastfeeding duration (\% months) } \\
\hline$<6$ & $189(24.8)$ \\
\hline$\geqslant 6$ & $574(75.2)$ \\
\hline \multicolumn{2}{|l|}{ Time of delivery before third survey (\% months) } \\
\hline $16-19$ & $263(34.5)$ \\
\hline 20 & $370(48.5)$ \\
\hline $21-24$ & $130(17.0)$ \\
\hline
\end{tabular}

acid consumption during pregnancy and the risk of wheeze or eczema in the offspring after multivariate adjustment.

After adjustment for potential confounders excluding maternal intake of vitamins $\mathrm{D}$ and $\mathrm{E}$ during pregnancy, inverse exposure-response associations between maternal intake of total fat, saturated fatty acids, monounsaturated fatty acids, n-3 polyunsaturated fatty acids and $\alpha$-linolenic acid during pregnancy and the risk of wheeze in infants were statistically significant whereas maternal intake of $\mathrm{n}-6$ polyunsaturated fatty acids and linoleic acid during pregnancy was not related to the risk of infantile eczema.

There were no evident relationships between maternal intake of meat and fish during pregnancy and the risk of wheeze or eczema in the offspring (table 4).
Table 2 Distribution of daily nutrients and food intake in 763 pregnant women at baseline*

\begin{tabular}{lc}
\hline Variable & Mean (SD) \\
\hline Daily nutrient intake & $7628.6(1924.1)$ \\
Total energy (kJ) & $61.6(10.8)$ \\
Total fat $(\mathrm{g})$ & $17.4(4.0)$ \\
Saturated fatty acids $(\mathrm{g})$ & $21.3(4.9)$ \\
Monounsaturated fatty acids $(\mathrm{g})$ & $2.4(0.7)$ \\
$\mathrm{n}-3$ Polyunsaturated fatty acids $(\mathrm{g})$ & $1.8(0.6)$ \\
$\alpha$-Linolenic acid $(\mathrm{g})$ & $0.17(0.11)$ \\
Eicosapentaenoic acid $(\mathrm{g})$ & $0.30(0.17)$ \\
Docosahexaenoic acid $(\mathrm{g})$ & $11.5(2.7)$ \\
$\mathrm{n}-6$ Polyunsaturated fatty acids $(\mathrm{g})$ & $11.3(2.6)$ \\
Linoleic acid $(\mathrm{g})$ & $0.14(0.04)$ \\
Arachidonic acid $(\mathrm{g})$ & $308.8(111.4)$ \\
Cholesterol $(\mathrm{mg})$ & $7.8(1.7)$ \\
Vitamin $\mathrm{E}(\mathrm{mg})$ & $6.2(3.7)$ \\
Vitamin D $(\mu \mathrm{g})$ & \\
Daily intake & $60.2(28.8)$ \\
Meat $(\mathrm{g})$ & $48.4(26.5)$ \\
Fish $(\mathrm{g})$ &
\end{tabular}

*Nutrient and food intake were adjusted for total energy intake using the residual method.

\section{DISCUSSION}

During immune maturation, naive Th0 cells are able to modulate the predominant expression of a Th1- or Th2-like cytokine pattern according to numerous eicosanoids and cytokines synthesised by antigen-presenting cells. ${ }^{16}$ Polyunsaturated fatty acids and their derivatives can influence the Th1:Th2 ratio. An intervention study among atopic pregnant Australian women showed that fish oil supplementation achieved a significant increase in $\mathrm{n}-3$ polyunsaturated fatty acids in neonatal erythrocyte membranes compared with the control group and that the neonatal in vitro interleukin 10 (IL10) response to cat allergen was significantly lower in the fish oil-supplemented group than in the control group. ${ }^{17}$ Other reports of that intervention study showed that IL13 levels in cord plasma and neonatal leukotriene $\mathrm{B}_{4}$ production by cord blood-derived neutrophils were significantly lower in infants from the intervention group than from the placebo group. ${ }^{18}{ }^{19} \mathrm{In}$ a randomised clinical trial of 528 pregnant Danish women, the risk of allergic asthma, atopic dermatitis or allergic rhinitis during the first 15-16 years of life was significantly lower in children whose mothers had received supplements of fish oil ( $2.7 \mathrm{~g}$ marine $\mathrm{n}-3$ polyunsaturated fatty acids per day) compared with those in the placebo group (olive oil), although the risk in children of mothers in the no-oil group was remarkably similar to the risk in children whose mothers had received fish oil. ${ }^{20}$ These findings agree with our results in relation to maternal intake of $\alpha$-linolenic acid and docosahexaenoic acid during pregnancy and the risk of infantile wheeze. The lack of association of wheeze in offspring with maternal eicosapentaenoic acid intake in the present study might to some extent be ascribed to the fact that docosahexaenoic acid is prefentially transferred to the fetus from the maternal circulation. ${ }^{21}$ A UK prospective study showed that the levels of 11 individual n-3 and $\mathrm{n}-6$ fatty acids in both cord blood red cells and maternal blood red cells obtained after 20 weeks of pregnancy were not related to the risk of infantile wheezing and eczema. ${ }^{22}$ Japanese people consume a large quantity of fish compared with people in Western countries, but the median value of daily marine origin $\mathrm{n}-3$ polyunsaturated fatty acid intake in the present study was $0.48 \mathrm{~g}$, which was much lower 


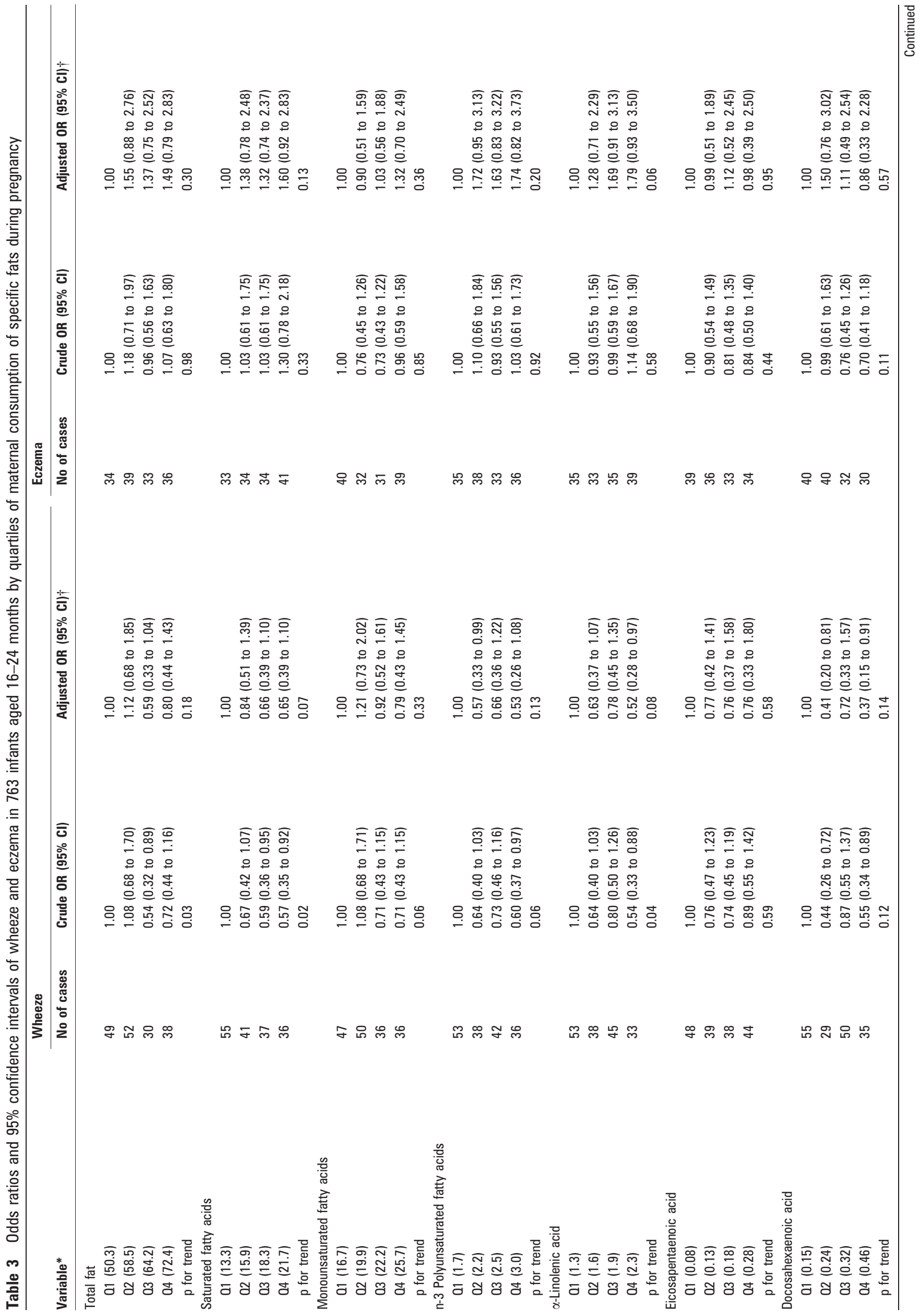




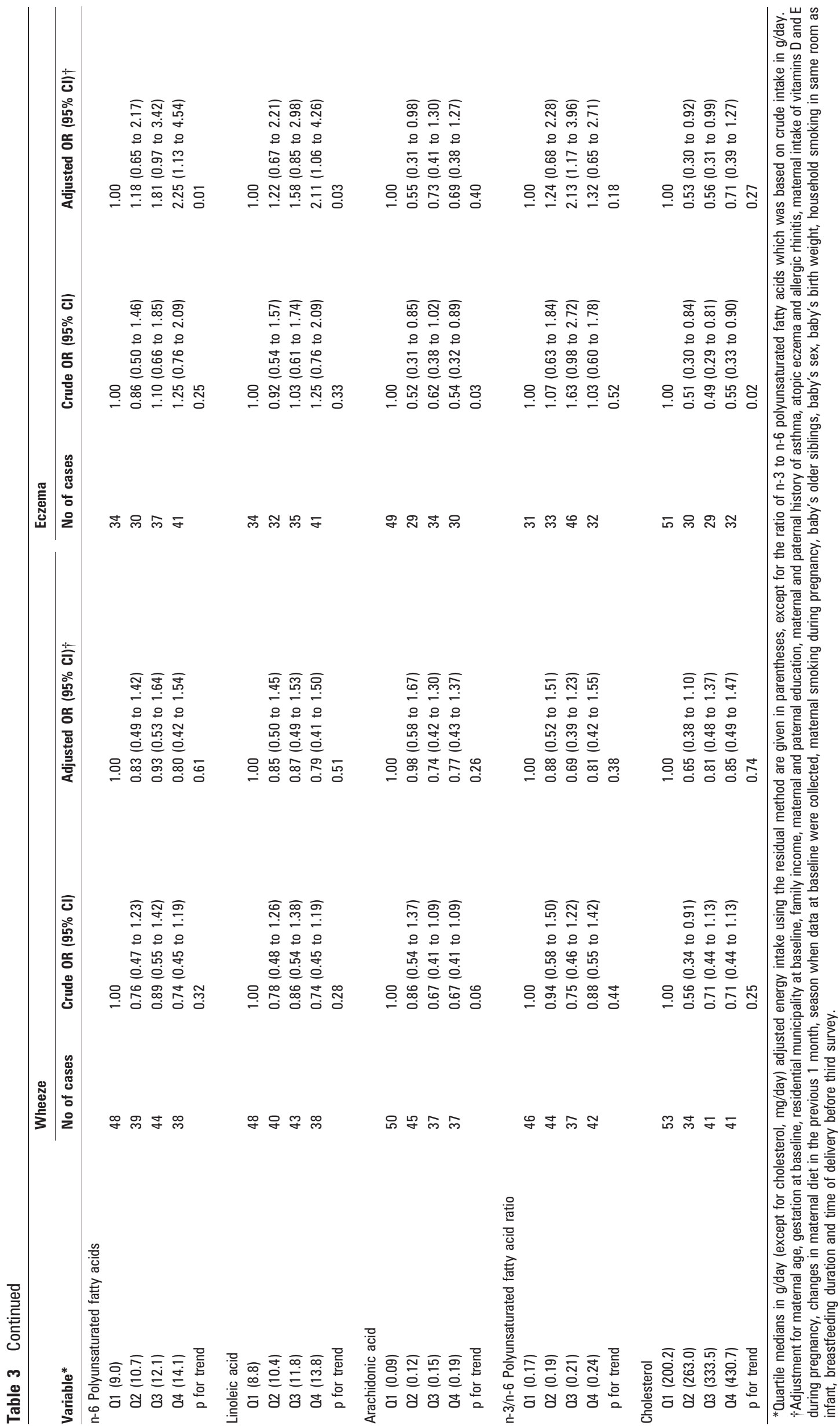




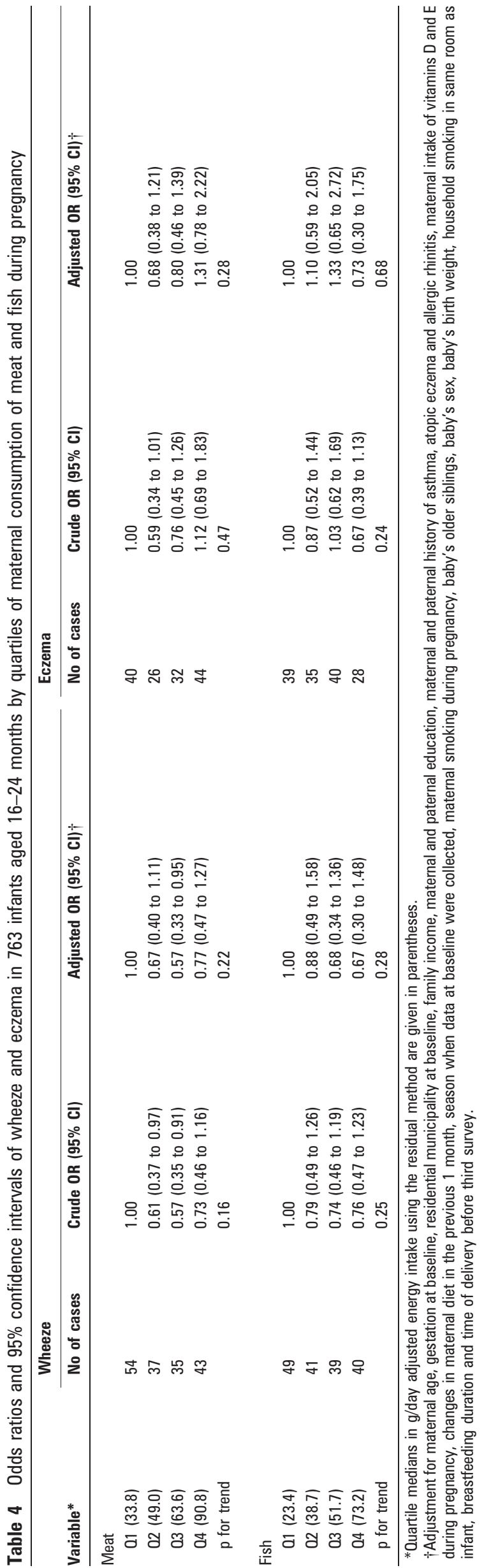

than the supplemented quantity in the Danish intervention study cited above. ${ }^{20} \mathrm{~A}$ clear protective relationship between maternal intake of marine origin $\mathrm{n}-3$ polyunsaturated fatty acids and the risk of infantile atopic eczema might be substantiated if consumption was much higher than in this study. The non-significant association of wheeze and eczema in the offspring with maternal fish intake in the current study could be due to unrecognised active agents in fish that interfered with the expected benefit to the offspring of maternal fish intake during pregnancy. For example, methylmercury and dioxins are accumulated in fish and shellfish through the marine food web. This hypothetical adverse effect might not be detected in Western populations where fish intake is low. ${ }^{2356}$

Linoleic acid is converted into arachidonic acid which enhances inflammatory processes via its derivatives prostaglan$\operatorname{din} \mathrm{E}_{2}$ and leukotriene $\mathrm{B}_{4}$. Our results regarding maternal intake of $\mathrm{n}-6$ polyunsaturated fatty acids and linoleic acid and infantile eczema might be reasonable. On the other hand, lipoxins derived from arachidonic acid decrease the synthesis of tumour necrosis factor $\alpha$ and leucocyte chemotaxis. ${ }^{16}$ Arachidonic acid, as well as docosahexaenoic acid, is essential to the placenta and the fetus. ${ }^{16}$ A US birth cohort study found that higher levels of cord blood eicosapentaenoic acid and arachidonic acid were associated with attenuation of cord blood lymphocyte proliferation and decreased secretion of interferon $\gamma$ in response to allergen stimulation, whereas higher levels of linoleic acid were correlated with increased allergen-stimulated IL13 levels. ${ }^{23}$ Given that the beneficial effect of arachidonic acid on fetal immune development counteracts the detrimental effect, the present findings on null associations between maternal intake of n-6 polyunsaturated fatty acids, linoleic acid and arachidonic acid during pregnancy and infantile wheeze also might be reasonable.

This study had methodological strengths. Study subjects were homogeneous with the same residential background. Extensive data on potential confounding factors were controlled for. In particular, maternal intake of vitamins $\mathrm{D}$ and $\mathrm{E}$ during pregnancy was the most important confounder. Such intake was inversely related to the risk of infantile wheeze and eczema. The prospective design was likely to reduce the possibility of recall bias. The definition of wheeze and eczema was based on the ISAAC questions. However, the outcomes under investigation were measured at varying ages between 16 and 24 months and, in addition, wheeze in infancy is known not to be a good predictor of asthma over the longer term. The consequence would have been an underestimation of values in our results.

Other weaknesses of the current study should be borne in mind. Our DHO could only approximate consumption. The consequence would bias the estimates of the observed association toward the null. The DHQ was designed to assess recent dietary intake (ie, for 1 month before completing the questionnaire). This disadvantage is likely to be alleviated after adjustment for the season when data were collected, however. Changes in diet in the past month were controlled for because pregnant women are likely to change their diet.

The follow-up rate was not good. Of the 1002 participants at baseline, 239 mother-child pairs did not take part in the third survey. There were no material differences between the 239 non-participants and the 763 participants in the third survey with regard to distribution of maternal age, maternal and paternal history of asthma, atopic eczema and allergic rhinitis, and maternal intake of total energy, monounsaturated fatty acids, $\mathrm{n}-3$ polyunsaturated fatty acids, $\mathrm{n}-6$ polyunsaturated fatty acids other than arachidonic acid, meat and fish. 
Compared with non-participants in the third survey, participants were less likely to report low family income and a low maternal and paternal educational level and were more likely to have a high intake of total fat, saturated fatty acids, cholesterol, arachidonic acid and vitamins $\mathrm{D}$ and $\mathrm{E}$. Moreover, at baseline the participation rate in Neyagawa City was only $17.2 \%$ and in other areas the participation rate could not be calculated because the exact number of eligible subjects among the sources from which participants were recruited was not available. Thus, the mother-child pairs in this study were probably not representative of Japanese mother-child pairs in the general population. In fact, educational levels were higher in the mothers in our study than in the general population. According to the 2000 population census of Japan, the proportions of women aged 30-34 years in Osaka Prefecture with years of education of $<13,13-14,15+$ and unknown were $49.2,32.3,13.6$ and $4.9 \%$, respectively. ${ }^{24}$ The present population might have had a greater awareness about health than the general population.

The current finding regarding the null association between maternal meat intake during pregnancy and infantile eczema was at variance with our previous results, showing a positive association between maternal meat intake during pregnancy and suspected atopic eczema in infants aged 3-4 months. The definition of suspected atopic eczema might have been crude. Of 58 infants identified with suspected atopic eczema, 25 (43.1\%) were classified as having eczema based on the ISAAC criteria in the third survey. In view of the multiple analyses and the $p$ values, it is possible that some or all of the findings in the current study and in our previous study occurred by chance.

Our results suggest that maternal intake of $\alpha$-linolenic acid and docosahexaenoic acid during pregnancy may be preventive against infantile wheeze whereas maternal intake of $n-6$ polyunsaturated fatty acids, especially linoleic acid, during pregnancy may increase the risk of childhood eczema. Further epidemiological investigations are required to ascertain whether the relationships observed in the current study are replicated in other populations.

Acknowledgements: The authors acknowledge the Neyagawa City Government, Hirakata City Government, Katano City Government, Shijonawate City Government, Kaizuka City Government, Takaishi City Government, Hannan City Government, Neyagawa City Medical Association, Hirakata City Medical Association, and the Kadoma City Medical Association for their valuable support.

Funding: Supported by a Grant-in-Aid for Scientific Research from the Ministry of Education, Culture, Sports, Science, and Technology and Health and Labour Sciences Research Grants, Research on Allergic Disease and Immunology from the Ministry of Health, Labour, and Welfare, Japan.

Competing interests: None.

Ethics approval: The ethics committee of the Osaka City University School of Medicine approved the OMCHS.

Provenance and peer review: Not commissioned; externally peer reviewed.

\section{REFERENCES}

1. Devereux G, Seaton A. Diet as a risk factor for atopy and asthma. J Allergy Clin Immunol 2005;115:1109-17.
2. Salam MT, Li YF, Langholz B, et al. Maternal fish consumption during pregnancy and risk of early childhood asthma. J Asthma 2005;42:513-8.

3. Romieu I, Torrent M, Garcia-Esteban R, et al. Maternal fish intake during pregnancy and atopy and asthma in infancy. Clin Exp Allergy 2007;37:518-25.

4. Willers SM, Wijga AH, Brunekreef B, et al. Maternal food consumption during pregnancy and the longitudinal development of childhood asthma. Am J Respir Crit Care Med 2008;178:124-31.

5. Sausenthaler S, Koletzko S, Schaaf B, et al. Maternal diet during pregnancy in relation to eczema and allergic sensitization in the offspring at 2 y of age. Am J Clin Nutr 2007;85:530-7.

6. Willers SM, Devereux G, Craig LCA, et al. Maternal food consumption during pregnancy and asthma, respiratory and atopic symptoms in 5-year-old children. Thorax 2007;62:773-9.

7. Saito K, Yokoyama T, Miyake $Y$, et al. Maternal meat and fat consumption during pregnancy and suspected atopic eczema in Japanese infants aged 3-4 months: The Osaka Maternal and Child Health Study. Pediatr Allergy Immunol 2009 (in press).

8. Miyake Y, Tanaka K, Sasaki S, et al. Breastfeeding and atopic eczema in Japanese infants: The Osaka Maternal and Child Health Study. Pediatr Allergy Immunol 2009;20:234-41.

9. Sasaki S, Yanagibori R, Amano K. Self-administered diet history questionnaire developed for health education: a relative validation of the test-version by comparison with 3-day diet record in women. J Epidemiol 1998;8:203-15.

10. Sasaki S, Ushio F, Amano K, et al. Serum biomarker-based validation of a selfadministered diet history questionnaire for Japanese subjects. J Nutr Sci Vitaminol 2000;46:285-96.

11. Science and Technology Agency. Standard tables of food composition in Japan 5th revised and enlarged ed. Tokyo: Printing Bureau of the Ministry of Finance, 2005 (in Japanese).

12. Science and Technology Agency. Standard tables of food composition in Japan: fatty acids section. 5th revised and enlarged ed. Tokyo: Printing Bureau of the Ministry of Finance, 2005 (in Japanese).

13. Willett W, Stampfer MJ. Total energy intake: implications for epidemiologic analyses. Am J Epidemiol 1986;124:17-27.

14. International Study of Asthma and Allergies in Childhood (ISAAC) Steering Committee. Worldwide variations in the prevalence of asthma symptoms: the International Study of Asthma and Allergies in Childhood (ISAAC). Eur Respir J 1998;12:315-35.

15. Williams H, Robertson C, Stewart A, et al. Worldwide variations in the prevalence of symptoms of atopic eczema in the international study of asthma and allergies in childhood. J Allergy Clin Immunol 1999;103:125-38.

16. Enke U, Seyfarth L, Schleussner E, et al. Impact of PUFA on early immune and fetal development. Br J Nutr 2008;100:1158-68.

17. Dunstan JA, Mori TA, Barden A, et al. Fish oil supplementation in pregnancy modifies neonatal allergen-specific immune responses and clinical outcomes in infants at high risk of atopy: a randomized, controlled trial. J Allergy Clin Immunol 2003;112:1178-84.

18. Dunstan JA, Mori TA, Barden A, et al. Maternal fish oil supplementation in pregnancy reduces interleukin-13 levels in cord blood of infants at high risk of atopy. Clin Exp Allergy 2003;33:442-8.

19. Prescott SL, Barden AE, Mori TA, et al. Maternal fish oil supplementation in pregnancy modifies neonatal leukotriene production by cord-blood-derived neutrophils. Clin Sci (Lond) 2007;113:409-16.

20. Olsen SF, Østerdal ML, Salvig JD, et al. Fish oil intake compared with olive oil intake in late pregnancy and asthma in the offspring: 16 y of registry-based follow-up from a randomized controlled trial. Am J Clin Nutr 2008;88:167-75.

21. Blümer $\mathbf{N}$, Renz $\mathbf{H}$. Consumption of omega3-fatty acids during perinatal life: role in immuno-modulation and allergy prevention. J Perinat Med 2007;35(Suppl): S12-8.

22. Newson RB, Shaheen SO, Henderson AJ, et al. Umbilical cord and maternal blood red cell fatty acids and early childhood wheezing and eczema. J Allergy Clin Immunol 2004;114:531-7.

23. Gold DR, Willwerth BM, Tantisira KG, et al. Associations of cord blood fatty acids with lymphocyte proliferation, IL-13, and IFN- $\gamma$. J Allergy Clin Immunol 2006;117:931-8.

24. Statistic Bureau, Ministry of Public Management, Home Affairs, Posts and Telecommunications. 2000 Population census of Japan, Volume 3-2-27: Labour force status of population, industry (major groups) of employed persons, and education. Osaka-fu. Tokyo: Statistic Bureau, Ministry of Public Management, Home Affairs, Posts and Telecommunications, 2002 (in Japanese). 